\begin{tabular}{|c|}
\hline $\begin{array}{c}\text { PRAMANA } \\
\text { journal of } \\
\text { physics }\end{array}$ \\
\hline
\end{tabular}

\title{
Microscopic neutron investigation of the Abrikosov state of high-temperature superconductors
}

\author{
JOHAN JUUL CHANG and JOEL MESOT* \\ Laboratory for Neutron Scattering, ETH Zurich and Paul Scherrer Institute, CH-5232 \\ Villigen-PSI, Switzerland \\ ${ }^{*}$ Corresponding author. E-mail: joel.mesot@psi.ch
}

\begin{abstract}
Using small angle neutron scattering we have been able to observe for the first time a well-defined vortex lattice (VL) structure both in the hole-doped LSCO and electron-doped NCCO superconductors. Our measurements on optimally doped LSCO reveal the existence of a magnetic field-induced phase transition from a hexagonal to a square coordination of the VL. Various scenarios to explain such phase transition are presented. In NCCO also a clear square VL could be detected, which is unexpectedly kept down to the lowest measurable magnetic fields.
\end{abstract}

Keywords. High-temperature superconductivity; vortex lattice structures; small angle neutron scattering.

PACS Nos 61.12.Ex; 74.72.Dn

\section{Introduction}

Apart from the unusual electronic and magnetic behaviour of the cuprate hightemperature superconductors (HTSC), experiments reveal a tremendously rich variety of mesoscopic phenomena associated with the flux vortices in the mixed state [1]. Because of their two-dimensional electronic structure, the HTSC are highly anisotropic. The anisotropy is characterized by the ratio $\gamma=\lambda_{\perp} / \lambda_{\|}$, where $\lambda_{\perp}, \lambda_{\|}$ are the superconducting penetration depths for currents flowing perpendicular and parallel to the two-dimensional $\mathrm{CuO}_{2}$ planes. In $\mathrm{La}_{2-x} \mathrm{Sr}_{x} \mathrm{CuO}_{4+\delta}$ (LSCO) the degree of anisotropy $(\gamma=20$ for $x=0.15)$ lies between that of the $\mathrm{YBa}_{2} \mathrm{Cu}_{3} \mathrm{O}_{x}$ (YBCO) and $\mathrm{Bi}_{2} \mathrm{Sr}_{2} \mathrm{CaCu}_{2} \mathrm{O}_{8+x}$ materials [2]. The cuprates are also extreme typeII superconductors, indicated by the high value of the Ginzburg-Landau parameter $\kappa=\lambda / \xi$, where $\xi$ is the superconducting coherence length. In HTSC the combination of high transition temperature $T_{\mathrm{c}}$, high $\gamma$ and high $\kappa$ leads to exotic vortex behaviour, such as the phenomenon of vortex lattice (VL) melting (see figure 1 and ref. [1]). For a conventional (isotropic) pairing mechanism such anisotropic conduction properties can lead to distortions of the vortex lattice as the applied field is tilted towards the $\mathrm{CuO}_{2}$ planes, but the local coordination remains six-fold [3]. 


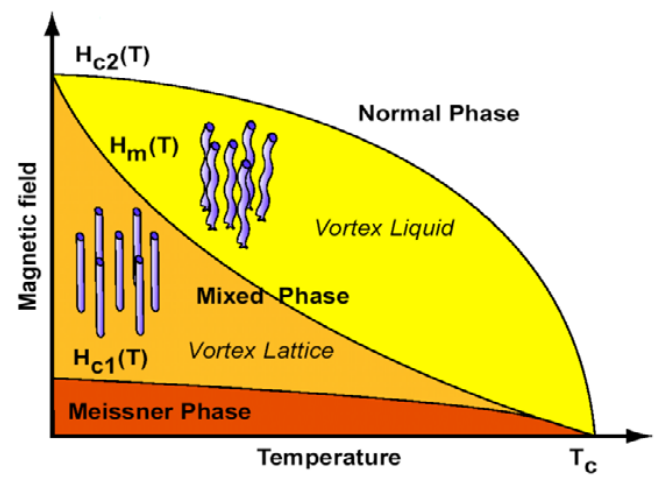

Figure 1. Schematic magnetic phase diagram of HTSC. For more details, see ref. [1].
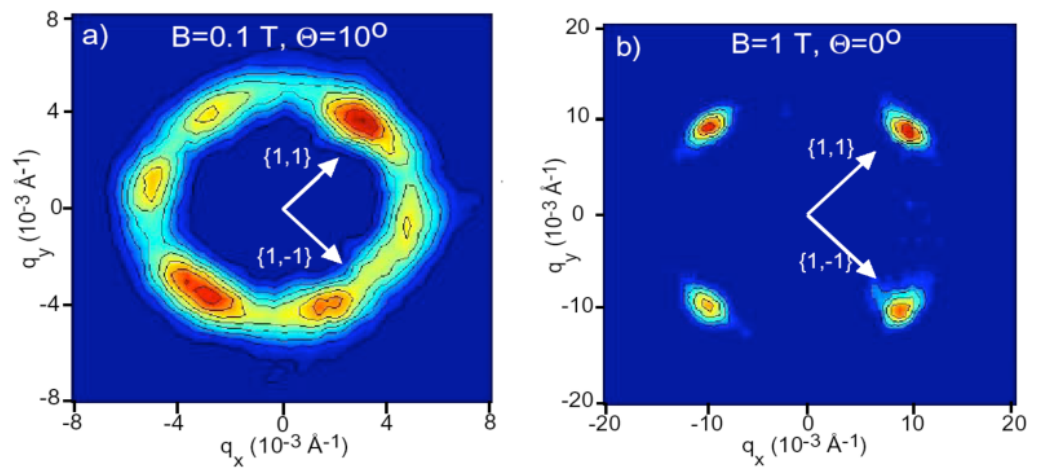

Figure 2. Vortex lattice measured in the superconducting phase of optimally-doped LSCO at (a) $H=0.1 \mathrm{~T}$ (hexagonal structure) and (b) $H=1 \mathrm{~T}$ (square structure) [4].

In the present paper, recent neutron results obtained in the Abrikosov phase of the hole-doped LSCO and electron-doped $\mathrm{Nd}_{2-x} \mathrm{Ce}_{x} \mathrm{CuO}_{4}$ (NCCO) HTSC as a function of doping are presented. The very high-quality LSCO crystals have been grown by the traveling solvent floating zone (TSFZ) technique by the group of Profs N Monomo, M Oda and M Ido of Hokkaido University, while the NCCO crystals were grown by the group of Prof. K Yamada, Tohoku University.

\section{Vortex lattice in optimally (hole)-doped LSCO}

Using small angle neutron scattering (SANS) we have succeeded, for the first time, to measure a well-ordered vortex lattice (VL) structure at all doping regimes of LSCO. In the optimally to overdoped regime a field-induced transition from hexagonal to square coordination is reported around $H=0.4 \mathrm{~T}$ (see figure 2 and ref. [4]) with the square lattice oriented along the anti-nodal direction of the d-wave 

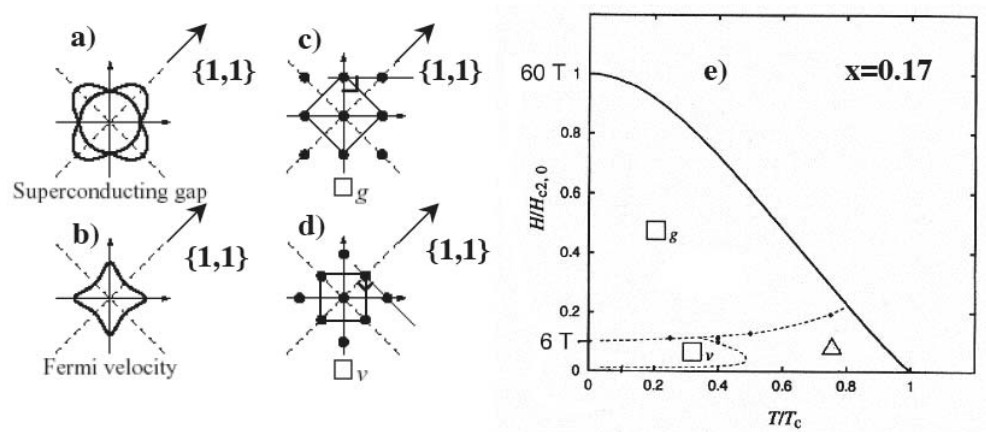

Figure 3. Both d-wave superconducting gap (panel a) and Fermi velocities (panel b) anisotropies will result in square lattices (panels $\mathrm{c}-\mathrm{d}$ ), but with different orientations with respect to the gap nodes. The resulting phase diagram taken from Nakai et al [8] for optimally-doped LSCO is given in panel e.

superconducting gap [5]. In a recent experiment, Brown et al [6] have observed a similar phase transition in the YBCO superconductor, however with two main differences: first, the critical field at which the transition occurs is at least an order of magnitude higher than in LSCO; second, the square VL in YBCO is oriented along the nodal direction of the d-wave gap function.

Various scenarios are able to explain such a hexagonal-to-square field-induced transition. It has been proposed that the importance of anisotropic vortex cores in a d-wave superconductor [7] would result in a square VL at high fields. Alternatively, coupling to other sources of anisotropy such as those provided by charge/stripe fluctuations or Fermi velocity anisotropies [8] should as well be considered. While the d-wave scenario favours a square VL aligned along the nodal direction (as observed in YBCO [6]), an anisotropy of the Fermi velocity would result in a VL aligned along the anti-nodal direction (as observed in LSCO [4]). These two competing scenarios are visualized in figure 3 .

In order to lift this apparent contradiction, Nakai et al [8], based on photoemission data [9] suggested that the observed orientation of the square lattice in optimally doped LSCO above $0.4 \mathrm{~T}$ is stabilized by the proximity of a van Hove singularity close to the Fermi level at the $(\pi, 0)$ anti-nodal point of the Brillouin zone. The extreme case of a van Hove singularity coinciding with the Fermi level, would result in a maximum anisotropy of the Fermi velocity along the nodal and anti-nodal directions. Such a strong anisotropy seems to be absent in YBCO, which would explain the different square lattice orientations. Nakai et al [8] furthermore predict that in LSCO, at high-enough fields $(H>6 \mathrm{~T})$, a second phase transition into a square VL oriented along the nodal direction should be observed due to the increasing importance of the d-wave anisotropy at high fields. So far our experiments realized up to $10.5 \mathrm{~T}[10,11]$ did not reveal such a transition, and experiments at even higher field values are necessary. 


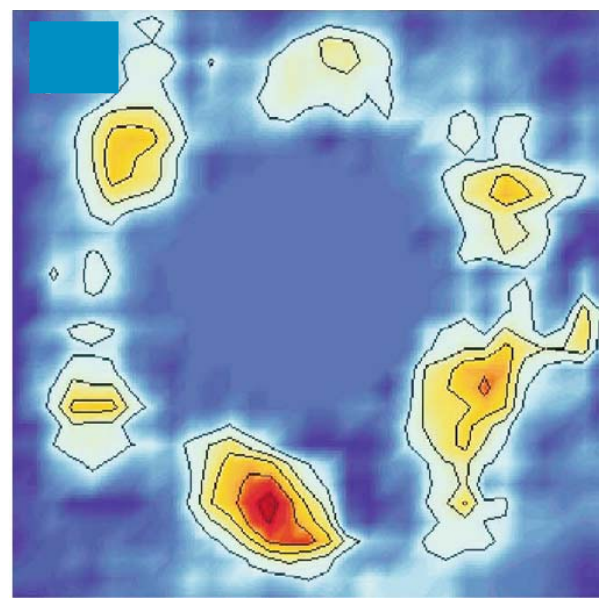

Figure 4. Small angle neutron diffraction pattern showing the existence of an ordered vortex phase at low fields $(150 \mathrm{Oe})$ and temperature $(6.2 \mathrm{~K})$. The intensity of this pattern rapidly falls as the field is increased towards the vortex-glass phase [14].

\section{Vortex lattice in underdoped LSCO}

The situation seems to be completely different in the underdoped regime of LSCO since a well-ordered hexagonal VL could be observed only at very low fields (see figure 4) [12]. By combining neutron scattering and muon spin rotation [12] it was concluded that the observed vanishing intensity with increasing field in the underdoped regime of LSCO is due to the a transition to a so-called vortex-glass state [13]. Such a state, exemplified in figure 5, is expected to occur when strong disorder is present. The transition from a well-ordered VL to a vortex-glass was studied carefully. In figure 6 we show the integrated intensity as a function of applied magnetic field in double logarithmic scale. The intensity decreases very fast with the applied magnetic field and follows approximately an $I \sim H^{-2}$ dependence over several decades. Eventually, the scattered signal from the VL becomes comparable with the overall background noise.

\section{Vortex lattice in electron-doped NCCO}

Successful measurements of a VL in the electron-doped $\mathrm{Nd}_{2-x} \mathrm{Ce}_{x} \mathrm{CuO}_{4}$ with $x=$ 0.15 [14] close to optimal doping could be performed.

Here again a square lattice (see figure 7 ) oriented along the $\mathrm{Cu}-\mathrm{O}$ axis was observed. However, the VL properties are markedly different from optimally-doped LSCO. First, the square lattice structure remained down to very low magnetic fields. Thus, a hexagonal-to-square transition as found in optimally-doped LSCO could not be observed. Second, the VL could only be observed for relatively low magnetic fields $(H<0.5 \mathrm{~T})$ in strong contrast to optimally-doped LSCO [10,11]. 


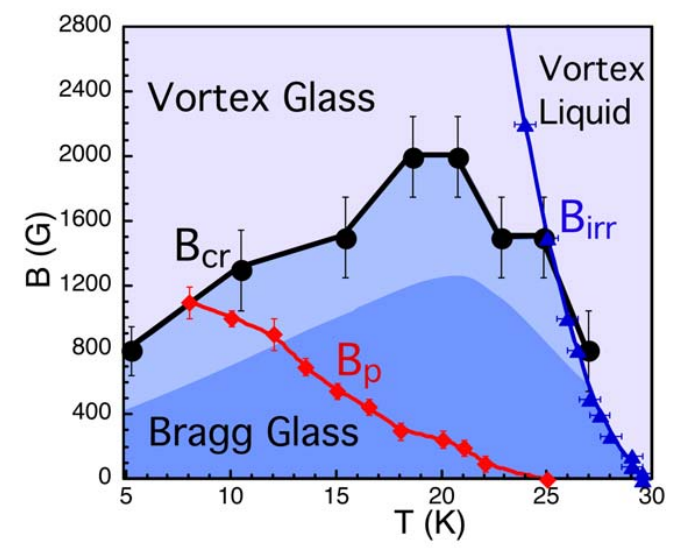

Figure 5. The magnetic phase diagram derived from the changes observed in the $\mu S R$ field distributions. $B_{\mathrm{cr}}$ indicates the onset of the broadening at high field, which is significant only below $25 \mathrm{~K}$, and should be considered as an upper limit for the Bragg-glass to vortex-glass transition. This uncertainty in the exact position of the transition is represented schematically by the shading below the line $B_{\mathrm{cr}}$. $B_{\text {irr }}$ indicates the irreversibility line as determined by bulk measurements of the field-cooled-zero-field-cooled (FC-ZFC) magnetization using a SQUID magnetometer. The position of the feature in the magnetization curves $B_{\text {on }}$ is also plotted [12].

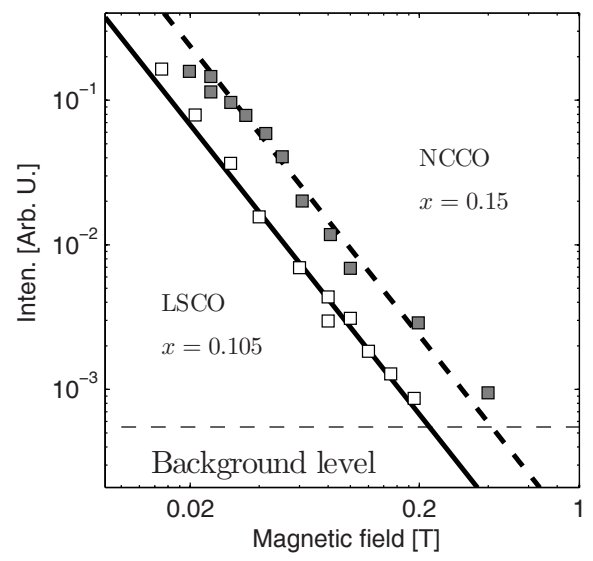

Figure 6. Integrated intensity as a function of applied magnetic field for LSCO $x=0.10$ and NCCO (dashed line). Notice that both axis are logarithmic. The solid and dashed line are $I \sim H^{2}[14]$.

The scattered intensity as a function of applied field is shown in figure 6 . As for underdoped LSCO the intensity follows an $I \sim H^{-2}$ dependence. This might suggest that the VL undergoes a transition to a vortex-glass as in underdoped LSCO. 

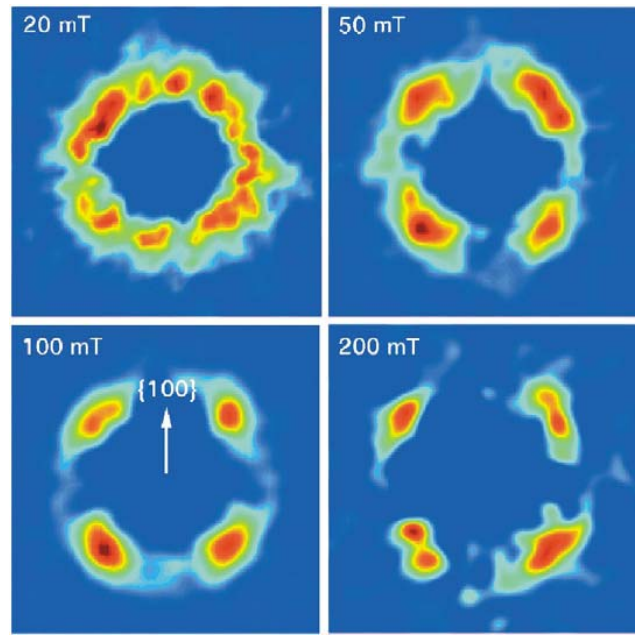

Figure 7. Vortex lattice in the electron-doped HTSC NCCO at various magnetic fields between 20 and $200 \mathrm{mT}$ [14].

\section{Acknowledgments}

This work would not have been possible without the intense collaboration of $\mathrm{R}$ Gilardi, J Kohlbrecher (ETH Zurich and Paul Scherrer Institute), U Divakar, A Drew, S Lee (Uni. St-Andrews), S Brown, E M Forgan (Uni. Birmingham), C Dewhurst, B Cubitt (Institute Laue-Langevin), N Monomo, M Oda and M Ido (Uni. Hokkaido) and K Yamada (Tohoku Uni.). The author also thanks K Machida (Okayama Uni.) and K Maki (Uni. S California) for fruitful discussions. This work is partially supported by the 21COE program from the ECSST Ministry of Japan, and by the Swiss NSF through grant No. 200020-105151 and NCCR MANEP.

\section{References}

[1] For a review, see G Blatter et al, Rev. Mod. Phys. 66, 1125 (1994)

[2] T Sasagawa et al, Phys. Rev. B61, 1610 (2000)

[3] S L Thiemann et al, Phys. Rev. B39, 11406 (1989)

M Yethiraj et al, Phys. Rev. Lett. 70, 857 (1993)

S T Johnson et al, Phys. Rev. Lett. 82, 2792 (1999)

M Yethiraj et al, Phys. Rev. Lett. 71, 3019 (1993)

[4] R Gilardi et al, Phys. Rev. Lett. 88, 217003 (2002)

[5] J Mesot et al, Phys. Rev. Lett. 83, 840 (1999)

[6] S P Brown et al, Phys. Rev. Lett. 96, 067004 (2004)

[7] A J Berlinsky et al, Phys. Rev. Lett. 75, 2200 (1995) Ji-Hai Xu, Yong Ren and Ting Chin-Sen, Phys. Rev. B53, R2991 (1996) J Shiraishi, M Kohmoto and K Maki, Phys. Rev. B59, 4497 (1999) M Ichioka, A Hasegawa and K Machida, Phys. Rev. B59, 8902 (1999)

[8] N Nakai et al, Phys. Rev. Lett. 89, 237004 (2002) 
[9] A Ino et al, Phys. Rev. B65, 094504 (2002)

[10] R Gilardi et al, Int. J. Mod. Phys. 17, 3411 (2003)

[11] J Chang et al, Phys. B. Condens. Matter 385-386, 35 (2006)

[12] U Divakar et al, Phys. Rev. Lett. 92, 237004 (2004)

[13] T Giamarchi and P Ledoussal, Phys. Rev. Lett. 72, 1530 (1994)

[14] R Gilardi et al, Phys. Rev. Lett. 93, 217001 (2004) 